\title{
A quick guide to safety and compati- bility of passive implants and devices in an MR environment
}

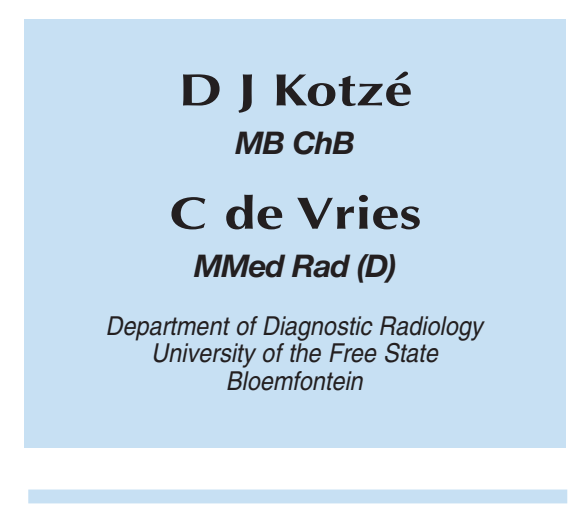

\section{Abstract}

An increasing number of patients with metal implants are being referred for magnetic resonance imaging (MRI) investigations. Implants and devices may be divided into two groups, namely active and passive. This article will focus on passive devices. A device is MR-safe when it is used in the MR environment, but presents no additional risk to the patient or other individuals, although the quality of diagnostic information may be affected. MR procedures may be contraindicated due to various interactions between the MR environment and medical devices, which include torque, translational force, heating, induced electrical currents, magnetic field interactions, artefacts, and mis- representation. Therefore, before deciding whether any object is MRsafe/compatible, the intended use and the possible retaining mechanisms must be considered.

\section{Introduction}

An ever-increasing number of patients are being referred for magnetic resonance imaging (MRI) investigations, with a subsequent increase in the number of patients presenting with old and new metal implants. The question arises whether it is safe for these patients to enter a magnetic resonance (MR) environment. After review of the literature we compiled this quick guide to safety and compatibility of implants and devices in an MR environment.

\section{General consid- erations}

Implants and devices may be divided into two groups, namely active (especially electronically activated devices, e.g. cochlear implants, implantable cardiac defibrillators, or any other activated device including ventilator and monitoring devices) and passive (clips, sutures, prostheses and any other device that serves its function without power supply). In this article the focus will be on passive devices.

When a device is MR-safe it means that when used in the MR environment the device presents no additional risk to the patient or other individuals, although the quality of diagnostic information may be affected. MRcompatible equipment is MR-safe and can be used in the MR environment with no significant effect on its operation or on the quality of diagnostic information. No metal is totally nonmagnetic or non-ferromagnetic, as all metals possess some degree of magnetism.

MR procedures may be contraindicated due to various interactions between the MR environment and medical devices, which include torque (product of axial force and the distance of the line of action from the axis) and translational force, both of which could cause possible movement or dislodgement of a ferromagnetic biomedical implant, material, device, or object. Other possible hazards and problems include heating, induced electrical currents (in materials that are conductors), magnetic field interactions (functional disruption of device), artefacts, and misrepresentation.

Translational attraction is assessed by using the deflection angle test, measured at the point of the 'highest spatial gradient' for the specific MR system. The deflection angle test central to MR safety testing for metallic implants and devices is as follows:

For deflections less than $45^{\circ}$ in the deflection angle test, the magnetically induced deflection force is less than the force of gravity on the implant. It 
implies that any risk imposed by the application of the magnetically induced force is no greater than any risk imposed by normal daily activity in the earth's gravitational field. There are no concerns about movement or dislodgement in people with implants and devices made from non-ferromagnetic or weakly ferromagnetic materials with a deflection angle between $0^{\circ}$ and $44^{\circ}$.

A torque value in any implant/ device that is less than that produced by normal daily activities (which include rapidly accelerating vehicles or amusement park rides) is assumed to be safe.

Translational attraction/deflection and torque could lead to movement or dislodging of ferromagnetic implants. This may cause discomfort or even serious injury to a patient. ${ }^{1}$ Translational attraction effects on external and implanted ferromagnetic objects in the immediate area around the MR system are usually responsible for possible dangers. Translational attraction is proportional to the static magnetic field strength, spatial gradient strength, and mass, shape and magnetic susceptibility of the object.

Deflection angles and magnetic field interactions of an implant can differ significantly between long- and short-bore MR. They are usually much higher in a short-bore. Higher magnetic field strength MR systems are rapidly increasing worldwide. Previously investigations were performed with magnetic fields only up to $1.5 \mathrm{~T}$. Weakly ferromagnetic objects in lower field strengths may experience much more interaction in higher field strengths. According to Faraday's law any change in a magnet field could induce a current in a conductor. This conductor in the MR environment could be cable, jewellery, metal external fixation devices or even a patient's arms if he holds his hands together above his head. The current induces heat in the conductor and that could present as burns on the patient.

In order to evaluate whether a device is now safe or compatible in the MR environment we need to keep in mind the things that could happen to this device. In summary the relative risk of injury depends on:

- Ferromagnetic properties of the foreign body

- Geometry and dimensions of the object

- Strength of the static magnetic field

- Strength of the spatial gradient of the MR system

- Amount of force with which the object is fixed within the tissue (i.e. counter-force or retention force)

- Whether it is positioned in or adjacent to a particularly sensitive site (vital neural, vascular, or soft tissue including eyes).

\section{Specific passive devices}

\section{Heart valve prostheses and annuloplasty rings ${ }^{2-4}$}

Most exhibited no magnetic field interactions but at 3.0 Tesla the Carpentier-Edwards Physio Annuloplasty Ring, Mitral Model 4450, Edwards Lifesciences, Irvine, USA showed relatively minor magnetic field interactions. The actual magnetic field interactions exerted on this implant are minor compared with the force exerted by the beating heart (i.e. approximately $7.2 \mathrm{~N}$ ), therefore MR procedures at 3.0 Tesla are not considered to be hazardous.

\section{Penile implants $\mathrm{s}^{2,3}$}

Weakly ferromagnetic penile implants may cause discomfort. Several different penile implants have been tested for MR safety in association with 3.0 Tesla MR systems but only Duraphase and Omniphase demonstrated relatively minor or 'weak' magnetic field interactions. Most of the other penile implants are considered safe for patients.

\section{Sutures ${ }^{2,3}$}

Two types (Flexon suture and Steel suture, United States Surgical, North Haven, CT) showed minor deflection angles and torque at 3.0 Tesla. The use of these materials will, however, provide sufficient counter-force to prevent movement or dislodgment. None of the other evaluated sutures (without their needles) displayed magnetic field interactions and appear to be safe at 3.0 Tesla.

\section{Aneurysm clips ${ }^{1-3,5-9}$}

Certain types of intracranial aneurysm clips (e.g. those made from martensitic stainless steels such as 17$7 \mathrm{PH}$ or 405 stainless steel) are absolutely contraindicated in MR procedures because magnetically induced forces may be excessive and these clips may displace or dislodge resulting in serious injury or death. Non-ferromagnetic, non-magnetic or weakly ferromagnetic aneurysm clips (e.g. those made from Phynox, Elgiloy, austentitic stainless steels, titanium alloy, or commercially pure titanium) are safe for MR use. Only one known ferromagnetic aneurysm clip-related fatality has been reported in the peerreviewed literature. Injury due to the presence of an aneurysm clip made from a non-ferromagnetic or weakly 
ferromagnetic material has never been reported. There have been cases where patients with ferromagnetic aneurysm clips (deduced from the extent of the artefact seen during MR imaging) have undergone MR procedures without any injuries. The deflection angle test and some form of evaluation of torque are probably the most appropriate means of determining whether a specific aneurysm clip may present a hazard to a patient or individual in the MR environment.

Aneurysm clips that are considered safe for patients or individuals exposed up to an 8.0 Tesla MR system, with deflection angles less than $45^{\circ}$ (ASTM guideline) and relatively minor qualitative torque values were manufactured from: commercially pure titanium (Spetzler), Elgiloy (Sugita), titanium alloy (Yasargil, Model FE 750T), and MP35N (Sundt).

Shellock's investigations were highly specific to the types of intracranial aneurysm clips that underwent testing (model, shape, size, blade length, material, etc.). Studies indicated that in spite of long-term and/or multiple exposures to 1.5 Tesla MR systems there were no clinically significant changes in their magnetic properties.

\section{Jewellery}

Mild-to-moderate movement and/or displacement depending on the body piercing site and the ferromagnetic qualities (e.g. mass, degree of magnetic susceptibility, etc.) of the jewellery may cause uncomfortable sensations. There is also a theoretical possibility of MRI-related heating that could cause burns. Metallic bodypiercing jewellery should be removed prior to entering the MR environment. If metallic jewellery or piercing cannot be removed the patient should be informed about the potential risks and preferably cancel the procedure. If it is not possible to cancel, then some means of stabilisation (e.g. application of adhesive tape or bandage) should be used to prevent movement or displacement in the MR. To prevent contact with the underlying skin and prevent excessive heating, wrap gauze or tape to at least $1 \mathrm{~cm}$ thick around piercing jewellery made from conductive materials.

\section{Breast devices $^{3}$}

Breast tissue expanders constructed with magnetic ports allow for a more accurate detection of the injection site. Therefore, these devices are attracted to the static magnetic field of MR systems and may be uncomfortable or become dislodged, causing injury to a patient undergoing an MR procedure.

\section{Cardiovascular guidewire and catheters ${ }^{3}$}

Patients with cardiovascular catheters and accessories with internally or externally positioned conductive metallic components should not undergo MR procedures. Interventional MRI devices and catheters without metallic components were deemed safe and were not included in the overall ex vivo tests for MR safety. It should be noted that these catheters and accessories were evaluated for MR safety without being connected to monitoring equipment.

There is at least one report of a cardiovascular catheter that melted in a patient undergoing MR imaging. There has never been a report of an incident or injury related to retained cardiac pacing wires in association with an MR procedure.

\section{Coils, filters and stents $\mathbf{s}^{3,10-13}$}

Several of these displayed magnetic field interactions during exposure to an MR environment, although most are incorporated securely into the vessel wall due to tissue ingrowth at about 6 - 8 weeks after placement. Similar devices made from non-ferromagnetic materials, such as the LGM IVC filter (Vena Tech) used for caval interruption or the Wallstent biliary endoprosthesis (Schneider (USA), Inc.) used for treatment of biliary obstruction, are considered safe for patients undergoing MR procedures.

However, not all stents are safe: Gianturco stent (Cook), and the modified Gianturco stent (Song), made of stainless steel, displayed magnetic field interactions where the deflection angles were greater than $45^{\circ}$ exceeding the ASTM guideline. Retention by tissue ingrowth and stents with hooks or barbs (to prevent migration after placement) may prevent them from posing a substantial risk to an individual.

\section{Cranial fixation devices ${ }^{3,14}$}

The clamps used for the cranial bone flap fixation system showed no magnetic field interaction and little heating; therefore they seem to pose no risk to the patient in a $<1.5$ Tesla MR environment. Cranial and burr hole fixation implants and devices made from titanium have been tested safe at 3.0 Tesla.

\section{Dental devices ${ }^{3,15}$}

Many of the dental implants, devices, materials, and objects evaluated for ferromagnetic qualities exhibited measurable deflection forces, but 
only the ones that have magnetically activated components present a potential problem for patients during MR procedures. The other dental implants, devices and materials are held in place with sufficient counterforces to prevent them from causing problems by being moved or dislodged by exposure to MR systems operating at 1.5 Telsa or less.

\section{Contraceptive devices ${ }^{3,16}$}

Various devices are listed:

- Diaphragms contain metallic rings, but they are not a contraindication at 1.5 Tesla.

- ESSURE is a novel metallic implant for permanent female contraception (in the USA). This is a dynamically expanding micro-coil that is placed in the proximal section of the fallopian tube via a non-incisional technique. An intended benign tissue response that is local, fibrotic and occlusive results in tissue in-growth into the device. It is thus anchored into the fallopian tube. There are no magnetic field interactions, the highest temperature changes were $<+0.6^{\circ} \mathrm{C}$, and the induced electrical currents are minimal.

- The Lea shield is a silicon rubber intravaginal barrier.

- Intrauterine contraceptive devices (IUCDs) are usually made from non-metallic materials (e.g. plastic) or a combination of non-metallic and metallic materials. Copper is the metal most used in an IUCD without side-effect.

- The Mirena device is safe at all static magnetic field strengths.

\section{ECG electrodes ${ }^{3}$}

Some patients need monitoring during the MR procedure, especially if there is a deterioration of vital signs during the investigation. As MR-guided surgery and therapy are also growing, there is an increased need to monitor patients. Investigations that use the electrocardiogram (ECG) for the purpose of gating also require the proper acquisition of the appropriate physiological signal for accurate representation of the MR images. Patient safety and proper recording of the ECG in the MRI environment require specially developed ECG electrodes. Using them during MRI procedures protects the patient from potentially hazardous conditions and produces minimal MRI-related artifacts. Special fibre-optic ECG recording techniques may be used to prevent burns during MR procedures.

\section{Foley catheters ${ }^{2,3}$}

There are Foley catheters available with a temperature sensor. These should never be connected to the temperature monitor during the MR procedure because the equipment is not MR-compatible or safe.

\section{Cervical fixation devices ${ }^{2,3,17,18}$}

MR procedures should only be performed on patients with halo vests or cervical fixation devices made from non-ferromagnetic and non-conductive materials, that have little or no interaction with the electromagnetic fields generated by MR systems. Halo vests or cervical fixation devices may be constructed from either ferromagnetic, non-ferromagnetic, or a combination of metallic components and other materials. Although some commercially available halo vests or cervical fixation devices are composed entirely of non-ferromagnetic materials, there is always a theoretical hazard of inducing electrical current in the ring portion of any halo device made from conductive materials (Faraday's law of electromagnetic induction). The patient is also susceptible to possible burn or electrical injuries. Noteworthy vibration of metallic components of devices like the halo ring, vertical supports, vest bolts, etc. were observed during MR imaging.

\section{Haemostatic clips ${ }^{2,3}$}

In several studies at $1.5 \mathrm{~T}$ no static magnetic field attraction to the clips was observed. At 3.0T the Surgiclip spring made from carbon steel (United States Surgical, North Haven, USA) showed a deflection angle of $90^{\circ}$ and a qualitative torque of +4 . This implant is currently categorised as 'unsafe' at 3.0T even though the closing force may provide substantial counterforce to prevent it from being moved or dislodged. Most of these implants were manufactured from non-ferromagnetic materials such as tantalum, commercially pure titanium, and non-ferromagnetic forms of stainless steel. Some ligating, haemostatic, or other types of clips are made from biodegradable materials. Patients who have haemostatic vascular clips, other clips, fasteners, and staples as mentioned in Shellock's booklet should not be at risk for injury during MR procedures. There has never been a report of an injury to a patient associated with a haemostatic vascular clip, other type of clip, fastener, or staple in the MR environment. Patients with non-ferromagnetic versions of these implants may undergo MR procedures immediately after they are placed surgically. Patients with metallic carotid artery vascular clamps have been imaged using static magnetic fields ranging up to $1.5 \mathrm{~T}$ without experiencing any discomfort 
or neurological consequence, with the exception of the Poppen-Blaylock clamp.

A metallic marking clip, the Micromark, made from 316L stainless steel by Biopsys Medical (Irvine, CA), has been developed for percutaneous placement after stereotactic breast biopsy. MicroMark II Clip (316LVM stainless steel, Ethicon Endosurgery, Cincinnati, Ohio) has been tested for MR safety in 1.5 Tesla and 3.0 Tesla MR systems. The findings indicated that there were no magnetic field interactions associated with exposure to 1.5 Tesla and 3.0 Tesla MR systems or MRI-related heating. Owing to excessive ferromagnetism and the associated imaging artefacts that may limit or obscure the area of interest, most biopsy needles, markers and devices are not useful for MR-guided biopsy procedures.

\section{Otological implants, ${ }^{3,19}$}

The ferromagnetic McGee stapedectomy piston prosthesis is made from platinum and chromium-nickel alloy stainless steel. The manufacturer has recalled this particular otological implant and patients who received these devices should avoid MR procedures.

\section{Ocular implants ${ }^{3,20}$}

Beware of intra-ocular foreign bodies. A patient with a Fatio eyelid spring or round wire eyelid spring may experience discomfort but would probably not be injured as a result of exposure to the magnetic fields of an MR system. Patients have undergone MR procedures with eyelid wires after having a protective plastic covering placed around the globe along with a firmly applied eye patch. Although no such case has ever been reported, the
Troutman magnetic ocular implant and retinal tacks (made from martensitic stainless steel) may cause injuries to a patient during an MR procedure.

\section{Orthopaedic implants ${ }^{3,21}$}

Most orthopaedic implants, materials, and devices evaluated for ferromagnetism are made from non-ferromagnetic materials and therefore should be safe for patients undergoing MR procedures. Only the Perfix interference screw used for reconstruction of the anterior cruciate ligament has been found to be highly ferromagnetic. Because this interference screw is firmly imbedded in bone, it is held in place with sufficient force to counterbalance it and to prevent movement or dislodgement and should not be considered a contraindication to MR. However, it is preferable to use interference screws made from non- or weakly ferromagnetic materials.

\section{Pellets and bullets ${ }^{3,22}$}

The risk against benefit and the anatomical location must be considered when deciding to perform an MR procedure in a patient with pellets, bullets, shrapnel or any other ballistic object because it may be contaminated with ferromagnetic materials.

\section{Surgical instruments ${ }^{3,23,24}$}

Interventional MRI procedures include a wide spectrum of minimally invasive surgical and therapeutic techniques that include percutaneous biopsy (e.g. breast, bone, brain, abdominal), endoscopic surgery of the abdomen, spine, and sinuses, open-brain surgery, and MR-guided monitoring of thermal therapies (i.e. laser-induced, RF-induced, and cryomediated procedures). For interventional MRI procedures surgical instruments and devices must be MRcompatible or at least MR-safe. The key problem of the conventional instruments and devices made from metallic materials in association with interventional MRI procedures is primarily image related.

Other concerns are: unwanted movement due to magnetic field interactions, the missile effect, translational attraction, torque and heating generated by RF power deposition.

The lack of commercially available, MR-compatible medical devices and instruments has hampered the widespread implementation of MRguided procedures, mainly those involving the use of complicated instruments such as the endoscope. Endoscopy in combination with MR guidance may offer several advantages including a dramatic improvement in the visualisation and orientation of the endoscope, an ability to appreciate complex three-dimensional anatomy in immediate and remote anatomical areas, and a reduction in procedurerelated morbidity. The use of commercially available endoscopes constructed from ferromagnetic materials is restricted in the MR environment owing to the associated substantial magnetic field attraction and production of large imaging artefacts.

MR systems like conventional, open-architecture, or the doubledonut MR systems (specially designed) for MR-guided biopsy, therapeutic, and minimally invasive surgical procedures are important clinical applications. To support these interventions and procedures innovative design and construction of instruments and devices are needed. Weakly ferromagnetic, non-ferromagnetic or non-metallic materials are used to make special instruments for inter- 
ventional MR procedures. Metallic surgical instruments and other devices potentially pose hazards (e.g. missile effects) or cause other problems such as image distortion, obscuring the region of interest, affecting adequate visualisation of the abnormality, and preventing execution of the procedure.

Ceramic instruments have exceptionally good qualities for the MR environment because there is no magnetic field attraction, negligible heating, and no substantial image distortion, as determined by the ex vivo testing.

\section{Tattoos and permanent cosmetics ${ }^{3,25,26}$}

Permanent cosmetics like tattooed eyeliner and decorative tattoos may cause MR imaging artefacts and relatively minor, short-term cutaneous reactions. The presence of a permanent cosmetic or decorative tattoo should not prevent the MR examination, since diagnostic information of vital significance may affect management of the patient.

\section{Prostate seed implant $t^{3}$}

Prostate cancer is sometimes treated by low-level radiation via a titanium tube implant with graphite, lead and palladium, the Theraseed. Tests demonstrated safety in an MR environment of 1.5T.

\section{Transdermal patches ${ }^{3}$}

Transdermal delivery system with a metallic component must be removed prior to an MR procedure, as the possibility of burn injury exists. Apply a new patch on completion of the examination.

\section{Vascular access ports ${ }^{3}$}

Various implantable vascular access ports and catheters evaluated for compatibility with MR procedures showed no measurable attraction to the static magnetic fields of the MR systems used for testing. The forces were considered to be minor relative to the in vivo application of these implants. Accessories, like the infusion set and needles, showed measurable ferromagnetism, with the PORT-ACATH Needle (Deltec, Inc., St Paul, $\mathrm{MN}$ ) exceeding the recommended ASTM deflection angle safety guideline (i.e. greater than $45^{\circ}$ ). A small strip of adhesive tape is an effective counterbalance to the ferromagnetism. Verify that the specific device is in Shellock's List and is safe to enter an MR environment.

\section{Postoperative patients and MR procedures ${ }^{2,3,12,13}$}

A patient with a metallic object 'passive implant' (no electronically or magnetically activated component associated with the operation of the device) that is made from non-ferromagnetic material (e.g. titanium, titanium alloy, nitinol, etc.), may undergo an MR procedure immediately after implantation in an MR system operating at 1.5 Tesla or less. A waiting period of $6-8$ weeks after placement of other implants or devices that exhibit 'weakly magnetic' qualities (e.g. certain stents, atrial septal defect occluders, ventricular septal defect occluders, patent ductus arteriosus occluders) is recommended before performing an MR procedure or allowing the individual or patient to enter the MR environment at $<1.5$ Tesla. 'Weakly' ferromagnetic intravascular and intracavitary coils, stents, filters, and cardiac occluders become firmly incorporated into tissue $6-8$ weeks following placement. Retentive or counter-forces provided by tissue ingrowth, scarring, or granulation essentially serve to prevent these objects from presenting risks or hazards to patients or individuals in the MR environment. Rigidly fixed implants or devices that may be 'weakly magnetic', such as a bone screw, may be studied immediately after implantation.

\section{Unconscious and unrespon- sive patients}

Protect the hearing of all unconscious and unresponsive patients prior to being scanned. Halfway through the procedure all monitoring lead locations should be repositioned as patients may be unable to report an increase in tissue warmth or pending thermal injury.

\section{Conclusion}

Before deciding whether any object is MR-safe/compatible carefully consider the intended use and the possible retaining mechanisms like sutures, granulation or tissue ingrowth, thus preventing movement and the possible effect of heating of sensitive tissue.

This is a quick guide for passive devices. Active devices will be discussed in a later article. All MR rooms should have an up-to-date list of devices and a copy of F G Shellock's Pocket Guide to MR Procedures and Metallic Objects, which is about as complete as it gets. ${ }^{27}$ Another useful site is Shellock's MRI safety.com. ${ }^{28}$

\section{Acknowledgement}

F G Shellock's information has been reproduced with permission.

\section{References}

1. Shellock FG, Crues JV. Aneurysm clips: assessment of magnetic field interaction associated 
with a $0.2-\mathrm{T}$ extremity MR system. Radiology 1998; 208: 407-409.

2. Shellock FG, Curtis JS. MR imaging and biomedical implants, materials, and devices: an updated review. Radiology 1991; 180: 541-550.

3. Shellock FG, Morisoli S, Kanal E. MR procedures and biomedical implants, materials, and devices: 1993 update. Radiology 1993; 189: 587 599.

4. Shellock FG, Slimp G. Halo vest for cervical spine fixation during MR imaging. Am J Roentgenol 1990; 154: 631-632.

5. Becker RL, Norfray JF, Teitelbaum GP, et al. MR imaging in patients with intracranial aneurysm clips. Am J Neuroradiol 1988; 9: 885-889.

6. Shellock FG, Kanal E. Aneurysm clips: evaluation of MR imaging artifacts at 1.5 T. Radiology 1998; 209: 563-566.

7. Klucznik RP, Carrier DA, Pyka R, Haid RW Placement of a ferromagnetic intracerebral aneurysm clip in a magnetic field with a fatal outcome. Radiology 1993; 187: 855-856.

8. Kanal E, Borgstede JP, Barkovich AJ, et al. American College of Radiology White Paper on MR Safety. Am J Roentgenol 2002; 178: 1335 1347.

9. Shellock FG, Kanal E. Yasargil aneurysm clips: evaluation of interactions with a 1.5-T MR system. Radiology 1998; 207: 587-591.

10. Hennemeyer CT, Wicklow K, Feinberg DA, Derdeyn CP. In vitro evaluation of platinum Guglielmi detachable coils at $3 \mathrm{~T}$ with a porcine model: safety issues and artifacts. Radiology 2001; 219: 732-737.
11. Hug J, Nagel E, Bornstedt A, Schnackenburg B, Oswald H, Fleck E. Coronary arterial stents: safety and artifacts during MR imaging. Radiology 2000; 216: 781-787.

12. Liebman CE, Messersmith RN, Levin DN, Lu CT. MR imaging of inferior vena caval filters: safety and artifacts. Am J Roentgenol 1988; 150: 1174-1176.

13. Manke C, Nitz WR, Djavidani B, et al. MR imaging-guided stent placement in iliac arterial stenoses: a feasibility study. Radiology 2001; 219: 527-534.

14. Shellock FG, Shellock VJ. Cranial bone flap fixation clamps: compatibility at MR imaging. Radiology 1998; 207: 822-825.

15. Teitelbaum GP, Bradley WG Jr, Klein BD. MR imaging artifacts, ferromagnetism, and magnetic torque of intravascular filters, stents, and coils. Radiology 1988; 166: 657-664.

16. Shellock FG, Shellock VI. MRI Safety of cardiovascular implants: evaluation of ferromagnetism, heating, and artifacts. Radiology 2000; 214 $\mathrm{P} 19 \mathrm{H}$.

17. Duru F, Luechinger R, Candinas R. MR imaging in patients with cardiac pacemakers. Radiology 2001; 219: 856-858.

18. Malko JA, Hoffman JC Jr, Jarrett PJ. Eddy-current-induced artifacts caused by an 'MR-compatible' halo device. Radiology 1989; 173: 563 564.

19. Nogueira M, Shellock F. Otologic bioimplants: ex vivo assessment of ferromagnetism and artefacts at 1.5 T. Am J Roentgenol 1994; 163: 14721473 .
20. Albert DW, Olson KR, Parel JM, Hernandez E Lee W, Quencer R. Magnetic resonance imaging and retinal tacks. Arch Ophthalmol 1990; 108: 320-321.

21. Shellock FG, Crues JV. High-field-strength MR imaging and metallic bioimplants: an in vitro evaluation of deflection forces and temperature changes induced in large prostheses (Abstract). Radiology 1987; 165: 150.

22. Teitelbaum GP. Metallic ballistic fragments: MR imaging safety and artifacts. Radiology 1990; 177: 883 .

23. Jolesz FA. 1996 RSNA Eugene P Pendergrass New Horizons Lecture. Image-guided procedures and the operating room of the future. Radiology 1997; 204: 601-612.

24. Shellock FG. Compatibility of an endoscope designed for use in interventional MR imaging procedures. Am J Roentgenol 1998; 171: $1297-$ 1300.

25. Wagle WA, Smith M. Tattoo-induced skin burn during MR imaging. Am J Roentgenol 2000; 174: 1795.

26. Carr JJ. Danger in performing MR imaging on women who have tattooed eyeliner or similar types of permanent cosmetic injections. Am J Roentgenol 1995; 165: 1546-1547.

27. Shellock FG. Pocket Guide to MR Procedures and Metallic Objects. Update 1996. Philadelphia: Lippincott, 2001.

28. Shellock FG. MRI safety.com. 2001 (accessed February - April 2004). Available at: http//www.mrisafety.com/1 